\title{
Harnessing the Immune Regulatory Balance of Toll-like Receptor 9 Agonists in Cancer Immunotherapy.
}

\author{
Cui $\mathbf{J}^{1 *}$, Bai $\mathrm{L}^{1}$, Zhou $\mathbf{L}^{1}$, Chen $\mathrm{W}^{2}$ and Chen $\mathrm{J}^{3}$ \\ ${ }^{1}$ Department of Cancer Center, The First Hospital of Jilin University, Changchun, Jilin, China \\ ${ }^{2}$ ADC Biomedical Research Institute, 1919 University Avenue, Saint Paul, MN, USA \\ ${ }^{3}$ Institute of Translational Medicine, The First Hospital of Jilin University, Changchun, Jilin, China
}

\begin{abstract}
Toll-like receptor 9 (TLR9), a naturally existing immune regulatory site, not only takes part in enhancing anti-tumor immunity but also promoting the immunosuppressive environment of the growing tumor. TLR9 has shown its potential activity as a therapeutic target in cancer immunotherapy. However, the increased pro-tumor inflammation mediated by TLR9 should also be considered. Therefore, it is important to develop a better understanding of its regulatory mechanisms to improve the therapeutic efficacy. This review will discuss the mechanisms of immune homeostasis regulated by TLR9 signaling pathways, and also introduce the strategies of how to harness the immune regulatory balance by TLR9 agonists, CpG oligodeoxynucleotides, in cancer immunotherapies.
\end{abstract}

Keywords: Toll-like receptor 9, CpG oligodeoxynucleotides, Plasmacytoid dendritic cells, Tumor microenvironment, Immunotherapy.

\section{Introduction}

With improved understanding of the immune system, immunotherapies, such as immune checkpoint inhibitor and chimeric antigen receptor $\mathrm{T}$ cell therapies, have shown unprecedented efficacy in treating cancer [1]. The success of cancer immunotherapy reveals the power of host immunity on killing cancer cells and the feasibility of unleashing restraints on anti-tumor immunity. However, the immunosuppressive tumor microenvironment (TME) and the low immunogenicity of cancer cells restrict the therapeutic efficacy of cancer immunotherapies in a fraction of patients [2-4]. Therefore, deciphering the underlying mechanisms promoting the generation of an immunosuppressive TME is urgently needed to better harness host anti-tumor immunity. Toll-like receptors (TLRs) serve as a bridge between innate and adaptive immunity [5-7], and are naturally existing immune regulatory sites that not only take part in the enhanced anti-tumor immunity but also influence the immunosuppressive environment of the growing tumor [8,9]. TLR9, one of 13 TLR types, has been widely studied as target molecular in relation to anti-tumor therapies in clinical trials [10]. Chemically synthesized $\mathrm{CpG}$ oligodeoxynucleotides (ODNs) are confirmed TLR9 agonists that directly inhibit cancer cells, as well as induce anticancer immune responses, with acceptable toxicity $[11,12]$. Thus, TLR9 plays a key role in multiple anticancer effects mediated by $\mathrm{CpG}$ ODNs. Furthermore, $\mathrm{CpG}$ ODNs may be exploited as immunotherapeutics or immune adjuvants to improve the efficacy of current immunotherapies.

In this review, we discuss the results of available studies to develop an improved understanding of homeostasis of the TME and regulation of the immune system triggered by TLR9 agonists. Due to the pro-tumor inflammatory cytokines produced by $\mathrm{CpG}$ ODNs, we also discuss options to exaggerate the anticancer effects without causing excessive inflammation promoting the development of cancer in order to improve current immunotherapies.

\section{TLR9-mediated TME in Immunotherapies}

\section{Homeostasis of the TME}

The TME consists of stromal cells, inflammatory cells, extracellular matrices as well as tumor cells. The TME not only plays an important role in the processes of tumor initiation, progression, and metastasis, but also influences the e $\square$ cacy of anti-tumor therapies [1,13]. Homeostasis of the immune system is maintained by a balance between the suppressed and enhanced immune responses. However, the TME is different from the normal tissue milieu. Tumor cells and tumorinfiltrating immune cells usually suppress activation of the immune system through changes in the expression or secretion of immunosuppressive factors. This maintains homeostasis of the TME via immune-tolerance [14,15].

Under steady state conditions, cytokines secreted by tumor cells, and tumor-infiltrating immune cells, can restrain the activation and maturation of pDCs. Non-activated $\mathrm{pDCs}$ play a critical role in the development and maintenance of the TME through decreasing the secretion of perforin and granzyme by CD8+ T cells, suppressing natural killer (NK) cell-mediated cytotoxicity, and promoting the differentiation of CD4+ T cells into activated regulatory $\mathrm{T}$ cells (Tregs) $[16,17]$. For example, interleukin(IL)-6 interferes with the maturation of pDCs, IL-8 blocks the migration of pDCs into lymph nodes, IL-10 inhibits 
the further expansion of T-cells mediated by pDCs, and elevated expression of cyclooxygenase- 2 interferes with $\mathrm{pDC}$ functions in multiple ways [8].

Non-activated pDCs (such as pDCs within tumor-draining lymph nodes and tumor-infiltrating pDCs) express high levels of suppressive immunomodulators such as indoleamine 2,3dioxygenase (IDO). The increased expression of IDO in pDCs results in activation of stress-response kinase GCN2, which can further inhibit $\mathrm{T}$ cell proliferation and promote the differentiation of $\mathrm{CD} 4+\mathrm{T}$ cells into activated Tregs by inhibiting the activation of $\mathrm{mTORC} 2$ and Akt signaling [8,18-23]. Apart from its contribution to Treg activation, IDO can also alter the phenotype of macrophages and neighboring cells to increase the secretion of inhibitory cytokines such as IL-10 and transforming growth factor (TGF)- $\beta$. Moreover, these macrophages and neighboring cells can inhibit the production of the positive regulator, IL-12 by pDCs $[24,25]$. Thus, in the context of tumor progression, the TME exhibits a multifaceted ability to play a suppressive role in the immune balance.

Although checkpoint blockade therapies can improve endogenous immune responses against tumors significantly, immunosuppressive mechanisms reduce the curative effects in some patients. It is likely that the dynamic TME can avoid immune-mediated clearance when inhibiting a single signaling checkpoint due to compensatory enhanced immunosuppressive mechanisms [26,27]. Thus, it is difficult to improve immunotherapy by only enhancing the anti-tumor effects or inhibiting the immunosuppressive mechanisms at a single signaling checkpoint. Reprogramming the immune response would be more beneficial.

\section{Regulation of the TME by TLR9 agonists}

TLR9 participates in both positive and negative immune regulation, thereby maintaining homeostasis of the immune system. TLR9 agonists can strengthen the recruitment or activation of activated effector cells to tumor tissue and overturn the pDCs induced immunosuppression mechanisms simultaneously. In other words, $\mathrm{CpG}$ ODNs harness the immune regulatory balance in cancer immunotherapy, especially the mechanisms related to maturation of pDCs, is key to this understanding.

\section{Immunomodulatory effects of CpG ODNs}

pDCs, the only DCs expressing TLR9, play a key role in initiating TLR9-mediated immune responses and balancing the TLR9-triggered immune system. The maturation of $\mathrm{pDCs}$ is affected by the presence of some diseases such as autoimmune diseases, viral infections, and tumors, and can then influence and further shift the immune balance (Figure 1) [16,20,28,29]. In addition, $\mathrm{T}$ cell types mediated by activated $\mathrm{pDCs}$ determine the suppression or activation of immune surveillance in the TME.

Through the pathway of interferon regulatory factor-7 (IRF-7) that is expressed constitutively at high levels in pDCs, $\mathrm{CpG}$ ODNs can induce pDCs to secrete type I interferons (IFNs), promote $\mathrm{pDC}$ maturation, increase inflammatory cytokine secretion by innate immune cells, and further promote adaptive immune responses to enhance immunotherapeutic effects [16,30-32]. Furthermore, IFN- $\alpha$ can block angiogenesis to reduce tumor growth $[33,34]$. In response to stimulation by CpG ODNs, pDCs differentiate into mature DCs with upregulated major histocompatibility complexes and costimulatory molecules. Such mature DCs have an increased ability of antigen cross-presentation to $\mathrm{CD} 8+\mathrm{T}$ cells $[20,35,36]$, and promote T-cell survival, type $1 \mathrm{~T}$ helper cell (Th1) polarization, $\mathrm{CD} 8+\mathrm{T}$ cells activation, memory $\mathrm{T}$ cell differentiation, and enhance NK cell-mediated cytotoxicity and IFN- $\gamma$ production [37-49]. In this situation, the immunosuppression state can be overturned and the immune system can further exert an anti-tumor effect.

\section{Molecular regulation mechanism of CpG ODNs}

Taking TLR9 downstream signaling pathways into consideration, $\mathrm{CpG}$ ODNs can not only promote the secretion of type I IFNs but also the production of some proinflammatory cytokines, such as IL-6. Therefore, TLR9 may contribute to anti-tumor effects as well as tumor-promoting signals [40-43]. Due to the dichotomous role of TLR9, it is important to develop a better understanding of its signaling pathway and the regulatory mechanisms of downstream molecules. This may provide new approaches to improve the e $\square$ cacy of current immunotherapies and decrease tumorpromoting inflammatory effects.

According to current research, the expression of TLR9 can be divided into two parts. While full-length TLR9 is expressed predominantly on the cell membrane, multiple cleaved forms are expressed mainly in the endoplasmic reticulum of $\mathrm{pDCs}$ and B cells. TLR9 expressed on the surface of B-cell lymphocytes can inhibit the activation of endosomal TLR9 $[44,45]$.

When stimulated by CpG ODNs, TLR9 initiates the activation of a family of IL-1 receptor-associated kinases (IRAKs), including IRAK-1, $-2,-4$ and $-\mathrm{M}$, after inducing the recruitment of myeloid differentiation antigen 88 (MyD88). The sequential activation of IRAKs induces an interaction with tumor necrosis factor associated factor (TRAF)-6 resulting in activation of nuclear factor- $\mathrm{\kappa B}(\mathrm{NF}-\mathrm{\kappa B})$ and mitogen-activated protein kinase. This will cause the most important cellular responses of inflammation or apoptosis [46]. In addition, NF$\kappa \mathrm{B}$ activation can upregulate many tumor-promoting inflammatory cytokines. These cytokines will promote NF- $\mathrm{KB}$ activation in a positive feedback loop [41]. Therefore, identifying negative regulators of NF- $\mathrm{BB}$ activation, such as the over-expression of TRIAD3A, an E3 ubiquitin-protein ligase, may provide a target to avoid excessive inflammation promoting the development of cancer [47].

On the other hand, direct interactions among MyD88, TRAF-6, and IRF-7 are required in TLR9-mediated pDC activation. Furthermore, IRAK-1 and -4 can activate IRF-7 which is required for the production of IFN- $\alpha$ [48].Thus, inhibitors of these molecules may disrupt anti-cancer effects. For example, transmembrane protein single immunoglobulin interleukin-1related receptor can suppress TLR9 activation and enhance 
sensitivity to endotoxin shock [47]. Over-expression of MyD88 can cause the formation of MyD88 homodimers and inhibit downstream MyD88-dependent signaling pathways by preventing IRAK-1 phosphorylation through IRAK-4 [49,50]. Similarly, suppressor of cytokine signaling 1 can inhibit the TLR9 signaling pathway by suppressing IRAK-1 expression [51]. IRAK-M, dependent on TGF- $\beta$, is also a negative regulator of TLR signaling that can promote the evasion of host immune surveillance by upregulating the rate of immunosuppressive M2 phenotype macrophages [52,53]. And IRAKs are deubiquitylated by A20 which blocks TLR9 signaling [54]. In addition, the interaction of p47 (phox) with TRAF4, which inhibits TRAF-6, plays a suppressive role in TLR9-mediated signaling [55].

Overall, owing to the biphasic modulation of TLR9 signals that affect the balance of the immune system, suppressing negative regulators related to the signals of IFN- $\alpha$ production, and simultaneously activating negative regulators related to protumor inflammatory signals may have anti-cancer effects. Although some molecules that negatively regulate TLR9 signaling have been found, their roles in tumor immunotherapy are just starting to emerge.

\section{Clinical applications of TLR9 agonists}

Available data indicate that TLR9 activation can harness the immune regulatory balance in cancer immunotherapy. There are some pre-clinical and clinical trials using CpG ODNs as an immune adjuvant to enhance the anti-tumor effects in some cancers as follows. Furthermore, $\mathrm{CpG}$ ODNs in combination therapies to promote $\mathrm{pDC}$ maturation may provide new insights into cancer treatment. However, TLR9 activation can also cause pro-inflammatory reactions. Thus, the heterogeneity of TLR9 signaling pathways in various cell types needs to be further considered and the impact of TLR9 agonists on antitumor responses should be evaluated.

\section{CpG ODNs as Immune adjuvants}

Although $\mathrm{CpG}$ ODNs have no direct pro-apoptotic effect on multiple myeloma cells, in some cases they can cause immune activation. When co-cultured with pDCs, CpG ODNs induce the maturation of pDCs that then secrete IFN- $\alpha$ and IFN- $\lambda$ which further induce G2-phase arrest in MM cells [56,57]. Besides, CpG ODNs can not only induce pDC maturation but also activate $\mathrm{NK}$ cells. Intravenous administration of $\mathrm{CpG}$ 7909 at doses of 0.01 to $0.64 \mathrm{mg} / \mathrm{kg}$ three times a week activates NK cells in patients with refractory non-Hodgkin lymphoma [58]. Because the activation of $\mathrm{pDCs}$ by $\mathrm{CpG}$ ODNs can enhance their antigen presentation activities, combination therapies with vaccines may improve the outcome in tumor patients. However, despite the fact that $\mathrm{CpG}$ ODNs treated with a vaccine-derived peptide can improve the production of peptide-specific $\mathrm{T}$ cells as well as cytokines (such as IFN- $\alpha$, TNF- $\alpha$, and IL-2) in vitro, there is no significant difference in the clinical outcomes between experimental and control group patients when comparing median progression-free and overall survival times with peptide vaccines including Melan-A/MART-1 peptide, gp100, MelQbG10, ISA-51, MAGE-A3, and Wilms' Tumor nuclear protein 1 [59-61]. Nevertheless, CpG ODNs can improve the killing effects of cytotoxic immune cells on tumor cells compared with using other therapies alone.

Many studies have shown that promoting the delivery of $\mathrm{CpG}$ ODNs to the tumor site may improve the anti-tumor effects. For example, some researchers found that intra- or peritumoral injection increased the infiltration of myeloid-derived suppressor cells, which will enhance the generation and activity of M1 macrophages. In that circumstance, the tumoricidal activity of M1 macrophages, and localization and the antigen-presenting ability of pDCs, would also be increased. Thus, compared to systemic injection of $\mathrm{CpG}$ ODNs, intra- or peri-tumoral injection was more effective $[62,63]$. Furthermore, compared with systemic administration, intrapulmonary delivery of $\mathrm{CpG}$ ODNs in non-small cell lung cancer decreased Treg- and M2 macrophage-mediated immunosuppression, increased the number of M1 macrophages, and prompted CD4+ $\mathrm{T}$ cells to differentiate into CD8+ T and Th1 cells [64].

\section{CpG ODNs combined with other therapies}

Chemotherapy, radiotherapy, and targeted therapy have shown powerful therapeutic effects on tumors. Combination therapies with CpG ODNs may improve these effects by activating immune cells. For example, combined therapy of CpG ODNs with cyclophosphamide (CTX) showed better outcomes inpatients with lymphoma, compared with CpG ODN or CTX therapy alone. Moreover, CTX inhibited the infiltration of Tregs into tumor sites, which enhanced the Th1 and cytotoxic lymphocyte responses [65]. Similar synergistic effects have been discovered in unresectable stage III and IV melanoma, advanced non-small cell lung cancer, and glioma [66-68].

Otherwise, $\mathrm{CpG}$ ODNs combined with radiotherapy result in a high complete tumor remission rate by increasing tumorreactive memory $\mathrm{CD} 8+\mathrm{T}$ cells, protecting normal $\mathrm{B}$ cells from irradiation-induced cell death, and enhancing M1 macrophage viability [69-71]. Additionally, in a phase I/II study, CpGbased in situ tumor vaccination improved radiotherapy outcomes with a high complete tumor remission rate, and induced systemic anti-lymphoma clinical responses by exerting direct effects on human malignant $\mathrm{B}$ cells and increasing tumor-reactive memory CD8 $+\mathrm{T}$ cells [72]. When combined with targeted therapies, CpG ODNs can increase the e $\square$ cacy of rituximab by promoting the activation and expansion of $\mathrm{Fc}$ receptor-bearing NK cells. The upregulated CD20 expression mediated by $\mathrm{CpG}$ ODNs might further promote the killing effects of rituximab [58,73].

\section{Perspective}

Despite the fact that TLR9 agonists have shown favorable activity in many clinical trials, the results of several trials are disappointing. In particular, clinical trials in recurrent or metastatic squamous cell carcinoma of the head and neck [74], and in non-small cell lung cancer [12], have yielded poor results. The magnitude and duration of responses to immunotherapies are affected by the tumor, the host, and the microenvironment. Because of these discouraging results, the 
application of $\mathrm{CpG}$ ODNs should be individualized, and more attention should be paid to the CpG ODN type, the treatment time point, and the application strategy of CpG ODN therapy. Furthermore, the heterogeneity of TLR9 expression, the distinctive responses in different cancers, and the influence of factors as well as mechanisms involved in immune homeostasis should be considered in cancer immunotherapies.

Although TLR9 activation can harness the immune regulatory balance in cancer immunotherapy, the increased pro-tumor inflammation and the over production of type I IFNs with the risk of causing autoimmune disease mediated by TLR9 should also be considered. By exploring the mechanism of $\mathrm{pDC}$ maturation, clarifying pivotal molecules that promote $\mathrm{pDC}$ maturation will help enhance $\mathrm{CpG}$ ODN-induced anti-tumor effects, in order to perform an optimal cancer immunotherapy with limiting tumor-promoting inflammatory effects and enhancing cytotoxic lymphocyte responses related signals without causing autoimmune reaction.

The comprehensive control of the immunosuppressive TME will significantly increase the effectiveness of current immunotherapies. As the concept of "cocktail therapy" has evolved [75], studies show that TME can sensitize cancer cells to DNA-damaging chemotherapies [76], cancer radiotherapy may be immunogenic [77], and antibody-CpG conjugates can improve immune stimulatory activity [78]. Therefore, combination therapies will become a new trend and the complexity of identifying optimal dosing levels and schedules for each component should also be taken into account, with better harnessing the immune regulatory balance of Toll-like receptor 9 agonists in cancer immunotherapy.

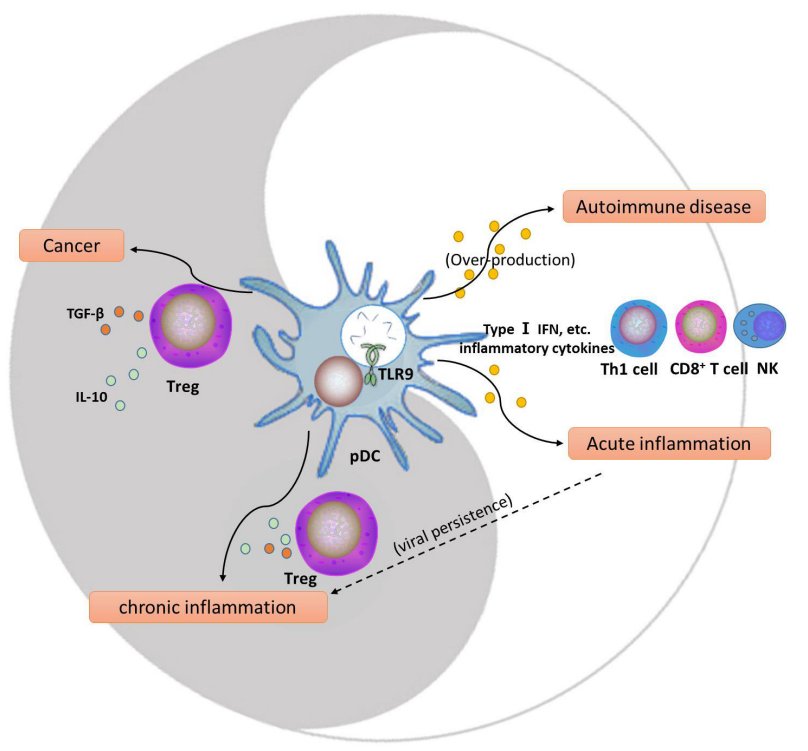

Figure 1. Toll-like receptor 9 agonists in cancer immunotherapy.

\section{Roles of Toll-like Receptor (TLR) 9 in Disease Status}

TLR9 plays a critical role in homeostasis of the immune system. Plasmacytoid dendritic cell (pDC) maturation shifts the balance from immunosuppression (dark segment) to immunoactivation (light segment). For example, in autoimmune diseases and acute inflammation, TLR9 agonists can induce mature pDCs to secrete inflammatory cytokines (such as type I IFNs) that further enhance the positive immune effects to promote type $1 \mathrm{~T}$ helper (Th1) cell and cytotoxic lymphocyte responses. On the other hand, pDCs may be deleted or become hypo-responsive to secrete type I IFNs, and promote $\mathrm{CD} 4+\mathrm{T}$ cells to differentiate into activated Tregs in chronic inflammation and cancer.

\section{List of Abbreviations}

TLR: Toll-like receptor; ODNs: oligodeoxynucleotides; TME: Tumor Microenvironment; IFNs: Interferons; pDCs: Plasmacytoid Dendritic Cells; Tregs: Regulatory T Cells; IL: Interleukin; IRF-7: Interferon Regulatory Factor-7; NK: Natural Killer Cell; IDO: Indoleamine 2,3-Dioxygenase; TGF: Transforming Growth Factor; MyD88: Myeloid Differentiation Antigen 88; IRAKs: IL-1 Receptor-Associated Kinases; NF$\kappa B$ : Nuclear Factor- $\kappa$ B; Th1: Type 1 T Helper; TRAF: Tumor Necrosis Factor Associated Factor; TRIF: Toll/IL-1 ReceptorDomain Containing Adaptor Protein Inducing IFN- $\beta$.

\section{Declarations}

\section{Ethics approval and consent to participate}

Not applicable.

\section{Consent for publication}

Not applicable.

\section{Availability of data and material}

The datasets supporting the conclusions of this article are included within the article and its additional files.

\section{Competing Interests}

The authors declare that they have no competing interests.

\section{Funding}

This work was supported by the National Natural Science Foundation of China (Grant 81672275 to JWC), the Ministry of Science and Technology of the People's Republic of China (Grant 2016YFC1303800 to JWC), the National Health and Family Planning Commission of the People's Republic of China (Grant ZX-07-C2016004 to JWC), the Key Laboratory Construction Project of Science and Technology Department (Grant 20170622011JC to JWC), the Industrial Research and Development Project of Development and Reform Commission of Jilin Province (Grant 2017C022 to JWC), and the Project of Development and Reform Commission of Jilin Province (Grant 2014N147 to JWC).

\section{Author Contributions}

LB carried out the primary literature search, drafted and revised the manuscript, and participated in discussions. LZ helped modify the manuscript. WC and JTC contributed to the 
coordination, and participated in discussions and literature searches. JWC carried out the design of the research and literature analysis, drafted and revised the manuscript, and participated in discussions. All authors read and approved the final version of the manuscript.

\section{Acknowledgments}

Not applicable.

\section{References}

1. Tang H, Qiao J, Fu YX. Immunotherapy and tumor microenvironment. Cancer Lett 2016; 370: 85-90.

2. Di Paolo JA, Huang T, Balazs $M$ et al. Specific Btk inhibition suppresses B cell-and myeloid cell-mediated arthritis. Nat Chem Biol 2011; 7: 41-50.

3. Berges C, Haberstock H, Fuchs D, et al. Proteasome inhibition suppresses essential immune functions of human CD4+ T cells. Immunol 2008; 124: 234-246.

4. Kim MG, Kim D, Suh SK, et al. Current status and regulatory perspective of chimeric antigen receptormodified T cell therapeutics. Arch Pharm Res 2016; 39: 437-452.

5. Chiron D, Bekeredjian-Ding I, Pellat-Deceunynck C, et al. Toll-like receptors: lessons to learn from normal and malignant human B cells. Blood 2008; 112: 2205-2213.

6. Sabroe I, Parker LC, Dower SK, et al. The role of TLR activation in inflammation. J Pathol 2008; 214: 126-135.

7. Agrawal S, Gupta S. TLR1/2, TLR7, and TLR9 signals directly activate human peripheral blood naive and memory B cell subsets to produce cytokines, chemokines, and hematopoietic growth factors. J Clin Immunol 2011; 31: 89-98.

8. Conroy H, Marshall NA, Mills KH. TLR ligand suppression or enhancement of Treg cells? A doubleedged sword in immunity to tumours. Oncogene 2008; 27 : 168-180.

9. Christensen SR, Shupe J, Nickerson K, et al. Toll-like receptor 7 and TLR9 dictate autoantibody specificity and have opposing inflammatory and regulatory roles in a murine model of lupus. Immunity 2006; 25: 417-428.

10. Krieg AM Development of TLR9 agonists for cancer therapy. Journal of clinical investigation 2007; 117: 1184-1194.

11. Bai L, Chen W, Chen J, et al. Heterogeneity of Toll-like receptor 9 signaling in $B$ cell malignancies and its potential therapeutic application. J Transl Med 2017; 15: 51.

12. Qin M, Li Y, Yang X, et al. Safety of Toll-like receptor 9 agonists: a systematic review and meta-analysis. Immunopharmacol Immunotoxicol 2014; 36: 251-260.

13. Klemm F, Joyce JA. Microenvironmental regulation of therapeutic response in cancer. Trends Cell Biol 2015; 25: 198-213.

14. Standiford TJ, Keshamouni VG. Breaking the tolerance for tumor: Targeting negative regulators of TLR signaling. Oncoimmunol 2012; 1: 340-345.
15. Munn DH, Mellor AL. IDO in the Tumor Microenvironment: Inflammation, Counter-Regulation, and Tolerance. Trends Immunol 2016; 37: 193-207.

16. Kadowaki N, Ho S, Antonenko S, et al. Subsets of human dendritic cell precursors express different toll-like receptors and respond to different microbial antigens. J Exp Med 2001, 194: 863-869.

17. Munn DH, Bronte V Immune suppressive mechanisms in the tumor microenvironment. Curr Opin Immunol 2016, 39: 1-6.

18. Munn DH, Sharma MD, Baban B, et al. GCN2 kinase in T cells mediates proliferative arrest and anergy induction in response to indoleamine 2,3-dioxygenase. Immunity 2005; 22: 633-642.

19. Sharma MD, Shinde R, McGaha TL, et al. The PTEN pathway in Tregs is a critical driver of the suppressive tumor microenvironment. Sci Adv 2015; 1: e1500845.

20. Lande R, Gilliet M Plasmacytoid dendritic cells: Key players in the initiation and regulation of immune responses. Ann N Y Acad Sci 2010; 1183: 89-103.

21. Liu Z, Gerner MY, Van Panhuys N, et al. Immune homeostasis enforced by co-localized effector and regulatory T cells. Nature 2015; 528: 225-230.

22. Sharma MD, Baban B, Chandler P, et al. Plasmacytoid dendritic cells from mouse tumor-draining lymph nodes directly activate mature Tregs via indoleamine 2,3dioxygenase. J Clin Invest 2007; 117: 2570-2582.

23. Wei S, Kryczek I, Zou L, et al. Plasmacytoid dendritic cells induce $\mathrm{CD} 8+$ regulatory $\mathrm{T}$ cells in human ovarian carcinoma. Cancer Res 2005; 65: 5020-5026.

24. Ravishankar B, Liu H, Shinde R, et al. The amino acid sensor GCN2 inhibits inflammatory responses to apoptotic cells promoting tolerance and suppressing systemic autoimmunity. Proc Natl Acad Sci USA 2015; 112: 10774-10779.

25. Ravishankar B, Shinde R, Liu H, et al. Marginal zone CD169+ macrophages coordinate apoptotic cell-driven cellular recruitment and tolerance. Proceedings of the National Academy of Sciences of the United States of America 2014; 111: 4215-4220.

26. Whiteside TL, Demaria S, Rodriguez-Ruiz ME, et al. Emerging opportunities and challenges in cancer immunotherapy. Clin Cancer Res 2016; 22: 1845-1855.

27. Moynihan KD, Opel CF, Szeto GL, et al. Eradication of large established tumors in mice by combination immunotherapy that engages innate and adaptive immune responses. Nat Med 2016; 22: 1402-1410.

28. Krieg AM, Vollmer $\mathrm{J}$ Toll-like receptors 7, 8, and 9: Linking innate immunity to autoimmunity. Immunol Rev 2007; 220: 251-269.

29. Means TK, Latz E, Hayashi F, et al. Human lupus autoantibody-DNA complexes activate DCs through cooperation of CD32 and TLR9. J Clin Invest 2005; 115: 407-417.

30. Guiducci C, Ghirelli C, Marloie-Provost MA, et al. PI3K is critical for the nuclear translocation of IRF-7 and type I IFN production by human plasmacytoid pre-dendritic cells 
Citation: Cui J, Bai L, Zhou L, Chen W, Chen J. Harnessing the Immune Regulatory Balance of Toll-like Receptor 9 Agonists in Cancer Immunotherapy. Immune System Disorder J 2017;1(2):20-26.

in response to TLR activation. J Exp Med 2008; 205: 315-322.

31. Kumagai Y, Takeuchi O, Akira S. TLR9 as a key receptor for the recognition of DNA. Adv Drug Deliv Rev 2008; 60: 795-804.

32. Honda K, Yanai H, Negishi H, et al. IRF-7 is the master regulator of type-I interferon-dependent immune responses. Nature 2005; 434: 772-777.

33. De Palma M, Mazzieri R, Politi LS, et al. Tumor-targeted interferon- $\alpha$ delivery by Tie2-expressing monocytes inhibits tumor growth and metastasis. Cancer Cell 2008; 14: 299-311.

34. Sidky YA, Borden EC. Inhibition of angiogenesis by interferons: effects on tumor-and lymphocyte-induced vascular responses. Cancer Res 1987; 47: 5155-5161.

35. Tang F, Du Q, Liu YJ. Plasmacytoid dendritic cells in antiviral immunity and autoimmunity. Sci China Life Sci 2010; 53: 172-182.

36. Le Bon A, Etchart N, Rossmann C, et al. Cross-priming of CD8+ $\mathrm{T}$ cells stimulated by virus-induced type I interferon. Nat immunol 2003; 4: 1009-1015.

37. Von Glehn F, Santos LM, Balashov KE. Plasmacytoid dendritic cells and immunotherapy in multiple sclerosis. Immunotherapy 2012; 4: 1053-1061.

38. Mantovani A, Romero P, Palucka AK, et al. Tumour immunity: effector response to tumour and role of the microenvironment. Lancet 2008; 371: 771-783.

39. Garaude J, Kent A, van Rooijen N, et al. Simultaneous targeting of toll- and nod-like receptors induces effective tumor-specific immune responses. Sci Transl Med 2012; 4: 120 ra116.

40. Pradere JP, Dapito DH, Schwabe RF. The Yin and Yang of toll-like receptors in cancer. Oncogene 2014, 33: 3485-3495.

41. Dajon M, Iribarren K, Cremer I. Toll-like receptor stimulation in cancer: A pro- and anti-tumor double-edged sword. Immunobiol 2017; 222:89-100.

42. Vesely MD, Kershaw MH, Schreiber RD, et al. Natural innate and adaptive immunity to cancer. Annu Rev immunol 2011; 29: 235-271.

43. Schreiber RD, Old LJ, Smyth MJ. Cancer immunoediting: Integrating immunity's roles in cancer suppression and promotion. Science 2011; 331: 1565-1570.

44. Tanaka J, Sugimoto K, Shiraki K, et al. Functional cell surface expression of toll-like receptor 9 promotes cell proliferation and survival in human hepatocellular carcinomas. Int J Oncol 2010; 37: 805-814.

45. Guerrier T, Pochard P, Lahiri A, et al. TLR9 expressed on plasma membrane acts as a negative regulator of human $\mathrm{B}$ cell response. J Autoimmun 2014; 51: 23-29.

46. Kawai T, Akira S. The role of pattern-recognition receptors in innate immunity: Update on toll-like receptors. Nat immunol 2010; 11: 373-384.

47. Wald D, Qin J, Zhao Z, et al. SIGIRR, a negative regulator of toll-like receptor-interleukin 1 receptor signaling. Nat Immunol 2003; 4: 920-927.
48. Fuchsberger M, Hochrein H, O'Keeffe M. Activation of plasmacytoid dendritic cells. Immunol Cell Biol 2005; 83: 571-577.

49. Burns K, Janssens S, Brissoni B, et al. Inhibition of interleukin 1 receptor/Toll-like receptor signaling through the alternatively spliced, short form of MyD88 is due to its failure to recruit IRAK-4. J Exp Med 2003; 197: 263-268.

50. Janssens S, Burns K, Tschopp J, et al. Regulation of interleukin-1- and lipopolysaccharide-induced NF-kappaB activation by alternative splicing of MyD88. Curr Biol 2002; 12: 467-471.

51. Kinjyo I, Hanada T, Inagaki-Ohara K, et al. SOCS1/JAB is a negative regulator of LPS-induced macrophage activation. Immunity 2002; 17: 583-591.

52. Standiford TJ, Kuick R, Bhan U, et al. TGF-beta-induced IRAK-M expression in tumor-associated macrophages regulates lung tumor growth. Oncogene 2011; 30: 2475-2484.

53. Kobayashi K, Hernandez LD, Galan JE, et al. IRAK-M is a negative regulator of Toll-like receptor signaling. Cell 2002; 110: 191-202.

54. Chuang TH, Ulevitch RJ TriadA. An E3 ubiquitin-protein ligase regulating Toll-like receptors. Nature Immunol 2004; 5: 495-502.

55. Takeshita F, Ishii KJ, Kobiyama K, et al. TRAF4 acts as a silencer in TLR-mediated signaling through the association with TRAF6 and TRIF. Eur J Immunol 2005; 35: 2477-2485.

56. Chauhan D, Singh AV, Brahmandam M, et al. Functional interaction of plasmacytoid dendritic cells with multiple myeloma cells: a therapeutic target. Cancer Cell 2009; 16: 309-323.

57. Cottini F, Anderson K. Novel therapeutic targets in multiple myeloma. Clin Adv Hematol Oncol 2015; 13: 236-248.

58. Leonard JP, Link BK, Emmanouilides C, et al. Phase I trial of toll-like receptor 9 agonist PF-3512676 with and following rituximab in patients with recurrent indolent and aggressive non-Hodgkin's lymphoma. Clin Cancer Res 2007; 13: 6168-6174.

59. Scheiermann J, Klinman DM. Clinical evaluation of $\mathrm{CpG}$ oligonucleotides as adjuvants for vaccines targeting infectious diseases and cancer. Vaccine 2014; 32: 6377-6389.

60. Baumgaertner P, Jandus C, Rivals JP, et al. Vaccinationinduced functional competence of circulating human tumor-specific CD8 T-cells. Int J Cancer 2012; 130: 2607-2617.

61. Vansteenkiste J, Zielinski M, Linder A, et al. Adjuvant MAGE-A3 immunotherapy in resected non-small-cell lung cancer: phase II randomized study results. J Clin Oncol 2013; 31: 2396-2403.

62. Betting DJ, Hurvitz SA, Steward KK, et al. Combination of cyclophosphamide, rituximab, and intratumoral $\mathrm{CpG}$ oligodeoxynucleotide successfully eradicates established B cell lymphoma. J Immunother 2012, 35: 534-543. 
63. Shirota H, Klinman DM. Use of CpG oligonucleotides for cancer immunotherapy and their effect on immunity in the tumor microenvironment. Immunother 2013; 5: 787-789.

64. Sato $T$, Shimosato $T$, Ueda $A$, et al. Intrapulmonary delivery of $\mathrm{CpG}$ microparticles eliminates lung tumors. Mol Cancer Ther 2015; 14: 2198-2205.

65. Li J, Song W, Czerwinski DK, et al. Lymphoma immunotherapy with $\mathrm{CpG}$ oligodeoxynucleotides requires TLR9 either in the host or in the tumor itself. Journal of immunology 2007; 179: 2493-2500.

66. Weber JS, Zarour H, Redman B, et al. Randomized phase 2/3 trial of CpG oligodeoxynucleotide PF-3512676 alone or with dacarbazine for patients with unresectable stage III and IV melanoma. Cancer 2009; 115: 3944-3954.

67. Manegold C, Gravenor D, Woytowitz D, et al. Randomized phase II trial of a toll-like receptor 9 agonist oligodeoxynucleotide, PF-3512676, in combination with first-line taxane plus platinum chemotherapy for advanced-stage non-small-cell lung cancer. J Clin Oncol 2008; 26: 3979-3986.

68. Jordan M, Waxman DJ. CpG-1826 immunotherapy potentiates chemotherapeutic and anti-tumor immune responses to metronomic cyclophosphamide in a preclinical glioma model. Cancer Lett 2016; 373: 88-96.

69. Mason KA, Neal R, Hunter $\mathrm{N}$, et al. CpG oligodeoxynucleotides are potent enhancers of radio- and chemoresponses of murine tumors. Radiotherapy and oncology. J Eur Society Ther Radiol Oncol 2006; 80: 192-198.

70. Milas L, Mason KA, Ariga $\mathrm{H}$, et al. $\mathrm{CpG}$ oligodeoxynucleotide enhances tumor response to radiation. Cancer Res 2004; 64: 5074-5077.

71. Mason KA, Ariga H, Neal R, et al. Targeting toll-like receptor 9 with $\mathrm{CpG}$ oligodeoxynucleotides enhances tumor response to fractionated radiotherapy. Clin Cancer Res 2005; 11: 361-369.

72. Brody JD, Ai WZ, Czerwinski DK, et al. In situ vaccination with a TLR9 agonist induces systemic lymphoma regression: A phase I/II study. J Clin Oncol 2010; 28: 4324-4332.

73. Betting DJ, Yamada RE, Kafi K, et al. Intratumoral but not systemic delivery of $\mathrm{CpG}$ oligodeoxynucleotide augments the efficacy of anti-CD20 monoclonal antibody therapy against B cell lymphoma. J Immunother 2009; 32: 622-631.

74. Guha M. Anticancer TLR agonists on the ropes. Nat Rev Drug Discov 2012; 11: 503-505.

75. Ledford H. Cocktails for cancer with a measure of immunotherapy. Nature 2016, 532: 162-164.

76. Sommariva M, De Cecco L, De Cesare M, et al. TLR9 agonists oppositely modulate DNA repair genes in tumor versus immune cells and enhance chemotherapy effects. Cancer Res 2011; 71: 6382-6390.

77. Gao C, Kozlowska A, Nechaev S, et al. TLR9 signaling in the tumor microenvironment initiates cancer recurrence after radiotherapy. Cancer Res 2013; 73: 7211-7221.

78. Li Z, Jang JK, Lechner MG, et al. Generation of tumortargeted antibody-CpG conjugates. J Immunol Methods 2013; 389: 45-51.

\section{*Correspondence to}

Cui J

Department of Cancer Center,

The First Hospital of Jilin University, Changchun, Jilin, China 